PROCEEDINGS OF THE

AMERICAN MATHEMATICAL SOCIETY

Volume 125, Number 10, October 1997, Pages 3075-3087

S 0002-9939(97)04020-3

\title{
THE AR-PROPERTY FOR ROBERTS' EXAMPLE OF A COMPACT CONVEX SET WITH NO EXTREME POINTS PART 1: GENERAL RESULT
}

\author{
NGUYEN TO NHU, JOSE M. R. SANJURJO, AND TRAN VAN AN \\ (Communicated by James West)
}

\begin{abstract}
We prove that the original compact convex set with no extreme points, constructed by Roberts (1977) is an absolute retract, therefore is homeomorphic to the Hilbert cube. Our proof consists of two parts. In this first part, we give a sufficient condition for a Roberts space to be an AR. In the second part of the paper, we shall apply this to show that the example of Roberts is an AR.
\end{abstract}

\section{INTRODUCTION}

In 1975 Roberts [R1] constructed a striking example of a compact convex set without any extreme points, giving a counter-example to the Krein-Milman theorem $[\mathrm{KM}]$ for non-locally convex linear metric spaces. After 1975 it was hoped that Roberts' example could be used as a counter-example to the following question (we call it the AR-problem): Is every convex set in a linear metric space an AR? ${ }^{1}$ See [BD], [G]. In fact, for about fifteen years, Roberts' example was the main target for attacking the AR-problem.

In [NT1], see also [N2], it was shown that every needle point space contains a compact convex AR-set with no extreme points. In particular, the spaces $L_{p}, 0 \leq$ $p<1$, as well as the linear metric space constructed originally by Roberts in [R1], contain compact convex AR-sets with no extreme points. Let us observe, however, that the results of [NT1] and [N2] do not apply to the original compact convex set, constructed in [R1]. In fact, the compact convex set, constructed originally by Roberts in [R1], has been distorted by the arguments in [NT1], [N2].

On the other hand, it was shown in [NT2] that all Roberts spaces have the fixed point property. (By a Roberts space we mean any compact convex set with no extreme points constructed by Roberts' method of needle point spaces; see [R1], $[\mathrm{R} 2],[\mathrm{KP}],[\mathrm{KPR}])$. However, the result of [NT2] does not say anything about the AR-property of Roberts spaces. Therefore, the AR-problem about Roberts' example [R1], posed in $[\mathrm{BD}]$, [G], has not yet been answered.

Received by the editors December 17, 1992 and, in revised form, April 1, 1996.

1991 Mathematics Subject Classification. Primary 54C55; Secondary 54D45.

Key words and phrases. Convex set, linear metric space, extreme point, absolute retract.

The first author was supported by the Complutense University of Madrid.

${ }^{1}$ In the non-compact case the AR-problem has been answered negatively by Cauty who recently constructed a $\sigma$-compact linear metric space which is not an AR; see Fund. Math. 146(1994), 85-99. For compact convex sets, the AR-problem, however, is still open.

(C)1997 American Mathematical Society 
The aim of this paper is to solve this problem. As a consequence of our result we obtain an affirmative answer to a problem of Dobrowolski and Mogilski; see [DM]. Our result shows that Roberts' example, while rather pathological in functional analysis, has a nice topological structure: In fact, it has the topology of the Hilbert cube, the simplest infinite dimensional compact object in topology. Our proof consists of two parts: In the first part (Sections 2-4), we establish a sufficient condition for a Roberts space to be an AR; see Theorem 2. Then, we apply our sufficient condition to prove in the second part (Sections 5-6) that the compact convex set with no extreme points, constructed by Roberts [R1], is an AR.

The fixed point property for all Roberts spaces was completely established. We have tried to obtain a similar answer for the AR-property of Roberts spaces. However, there are still some difficulties we have not yet found a way to overcome. It seems to the authors that the AR-property is somewhat harder than the fixed point property even for compact convex sets. In fact, our result shows that the AR-property of Roberts spaces is not easy, even in the very special case of Roberts' example [R1]. The AR-problem for Roberts spaces is still an important question. Further investigation to the AR-problem for Roberts spaces should be followed up.

Notation and conventions. By a linear metric space we mean a topological linear space $X$ which is metrizable. By Kakutani's theorem (see, for instance [Re]) there is an invariant metric $\rho$ on $X$. We denote $\|x\|=\rho(x, \theta)$, where $\theta$ is the zero element of $X$, which is called an F-norm.

By [Re, Theorem 1.2.2] we may assume that $\|$.$\| is monotone (or non-decreasing),$ that is,

$$
\|\lambda x\| \leq([|\lambda|]+1)\|x\| \text { for every } x \in X \text { and } \lambda \in \mathbb{R},
$$

where $[\alpha]$ denotes the greatest integer that is smaller than $\alpha$. In particular

$$
\|\lambda x\| \leq\|x\| \text { for every } x \in X \text { and } \lambda \in \mathbb{R} \text { with }|\lambda| \leq 1 .
$$

In this paper all linear metric spaces are assumed to be equipped with monotone $F$-norms. In particular, (2) will be used frequently throughout the paper.

Let $A$ be a subset of a linear metric space $X$. By conv $A$ we denote the convex hull of $A$ in $X$ and span $A$ denotes the linear subspace of $X$ spanned by $A$. We also use the following notation:

$$
A^{+}=\operatorname{conv}(A \cup\{\theta\}) ; \hat{A}=\operatorname{conv}\left(A^{+} \cup\left(-A^{+}\right)\right)=\operatorname{conv}(A \cup(-A) \cup\{\theta\}) ;
$$

and if $x, y \in X$ and $B \subset X$, we write

$$
\begin{aligned}
\|x-A\| & =\inf \{\|x-y\|: y \in A\} ; \\
\operatorname{dis}(A, B) & =\inf \{\|x-y\|: x \in A, y \in B\} ; \\
\operatorname{diam} A & =\sup \{\|x-y\| x, y \in A\} ; \\
{[x, y] } & =\{t x+(1-t) y: t \in[0,1]\} .
\end{aligned}
$$

If $A$ is a finite set, then card $A$ denotes the cardinality of $A$. Let $f: \mathbb{R} \rightarrow \mathbb{R}$ be a function; we denote $\operatorname{supp} f=\{t \in \mathbb{R}: f(t) \neq 0\}$.

\section{The AR-property for Roberts spaces}

The following theorem was established by Roberts in [R1]; see also [R2], [KPR, Proof of Theorem 9.4]. 
Theorem 1. Let $\left\{A_{n}\right\}$ be a sequence of finite sets of non-zero points in a complete linear metric space $X$ with the following properties:

$A_{0}$ consists of only one single point;

$$
\begin{aligned}
& A_{n+1}=\cup\left\{A\left(a, \varepsilon_{n+1}\right): a \in A_{n}\right\}, \text { where } \varepsilon_{n+1}=m(n)^{-1} 2^{-n-1}, \\
& m(n)=\text { card } A_{n}, \text { and } A\left(a, \varepsilon_{n+1}\right) \text { is a finite set, which is called } \\
& \text { an } \varepsilon_{n+1} \text {-needle set for } a, \text { that is, }
\end{aligned}
$$

$$
\begin{gathered}
\|b\|<\varepsilon_{n+1} \text { for every } b \in A\left(a, \varepsilon_{n+1}\right) ; \\
a \in \operatorname{conv} A\left(a, \varepsilon_{n+1}\right)
\end{gathered}
$$

For each $b \in A\left(a, \varepsilon_{n+1}\right)^{+}$, there exists an $\alpha \in[0,1]$ such that $\|b-\alpha a\|<\varepsilon_{n+1}$.

Denote $A=\overline{\bigcup_{n=o}^{\infty}\left(A_{n}\right)^{+}} \subset X ; C=\operatorname{conv}(A \cup(-A)) \subset X$. Then, $C$ is a compact convex set with no extreme points.

Remark 1. By (5-b), $\left\{\left(A_{n}\right)^{+}\right\}$and $\left\{\hat{A}_{n}\right\}$, see (3), are increasing sequences. Therefore, the set $A$ is convex, and by (5), $A$, and hence $C$, are compact. It is easy to see that

$$
C=\overline{\bigcup_{n=0}^{\infty} \hat{A}_{n}} \subset X .
$$

For every $a \in A_{n}$, let $A_{n+1}(a)=A\left(a, \varepsilon_{n+1}\right)$. For $k \geq n+2$, we define $A_{k}(a)$ by induction:

$$
\begin{gathered}
A_{k}(a)=\cup\left\{A\left(b, \varepsilon_{k}\right): b \in A_{k-1}(a)\right\} ; \\
C_{n}(a)=\overline{\bigcup_{k=1}^{\infty} \hat{A}_{n+k}(a)} \subset X .
\end{gathered}
$$

Observe that

$$
A_{n+k}=\cup\left\{A_{n+k}(a): a \in A_{n}\right\} .
$$

The following notation will be used frequently throughout the paper:

$$
A_{n}=\left\{a_{1}^{n}, \ldots, a_{m(n)}^{n}\right\}, \text { where } m(n)=\operatorname{card} A_{n} .
$$

In this part, we give a sufficient condition under which the compact convex set $C$ in Theorem 1 is an AR.

Theorem 2. $C$ is an $A R$ if the following conditions hold:

(i) For every $n \in \mathbb{N}$, if $x_{i} \in \operatorname{span} C_{n}\left(a_{i}^{n}\right) \backslash\{\theta\}, i=1, \ldots, m(n)$, then the set $\left\{x_{1}, \ldots, x_{m(n)}\right\}$ is linearly independent in $X$ (see (8) and (10)).

(ii) For every $k \in \mathbb{N}$, the sets $A_{k+1}(a)=A\left(a, \varepsilon_{k+1}\right), a \in A_{k}$, have the same cardinality (see (5)).

Remark 2. It follows from (i) that the union of (9) is disjoint. Therefore, Condition (ii) implies that card $A_{k+1}(a)=m(k)^{-1} m(k+1)$ for every $a \in A_{k}$.

At first we establish the following facts, which will be used in the proof of Theorem 2 . 
Lemma 1 [NT2]. Let $X$ be a linear metric space and let $a \in X$ be a non-zero point. Then, there is a retraction $r_{a}: X \rightarrow[-a, a]$ such that

$$
\left\|x-r_{a}(x)\right\| \leq 4\|x-[-a, a]\| \quad \text { for every } x \in X .
$$

Lemma 2. $\left\|x-\left[-a_{i}^{n}, a_{i}^{n}\right]\right\| \leq m(n)^{-1} 2^{-n+1}$ for every $x \in C_{n}\left(a_{i}^{n}\right)$ and $i=1, \ldots$, $m(n)$ (see (8), (10)).

Proof. Since $A_{n+1}\left(a_{i}^{n}\right)^{+}=A\left(a_{i}^{n} \varepsilon_{n+1}\right)^{+}$, by (5-c) for every $x \in A_{n+1}\left(a_{i}^{n}\right)^{+}$there exists an $\alpha \in[0,1]$ such that

$$
\left\|x-\alpha a_{i}^{n}\right\|<\varepsilon_{n+1}=m(n)^{-1} 2^{-n-1} .
$$

Therefore Lemma 2 holds for $x \in A_{n+1}\left(a_{i}^{n}\right)^{+}$. We shall prove by induction that, for every $x \in A_{n+k}\left(a_{i}^{n}\right)^{+}$,

$$
\left\|x-\left[\theta, a_{i}^{n}\right]\right\|<m(n)^{-1}\left(2^{-n-1}+2^{-n-2}+\cdots+2^{-n-k}\right) .
$$

In fact, let $A_{n+k}\left(a_{i}^{n}\right)=\left\{a_{1}, \ldots, a_{p}\right\}$. By Condition (ii) of Theorem $2, p$ does not depend on $i \in\{1, \ldots, m(n)\}$. From (5) and (7) we get

$$
A_{n+k+1}\left(a_{i}^{n}\right)=\cup_{j=1}^{p} A\left(a_{j}, \varepsilon_{n+k+1}\right) .
$$

So, for every $x \in A_{n+k+1}\left(a_{i}^{n}\right)^{+}$we have $x=\sum_{j=1}^{p} \lambda_{j} x_{j}$, where $x_{j} \in A\left(a_{j}, \varepsilon_{n+k+1}\right)^{+}$, $\lambda_{j} \geq 0, j=1, \ldots, p$, and $\sum_{j=1}^{p} \lambda_{j} \leq 1$; see (3). By (5-c), for every $j=1, \ldots, p$, there exists an $\alpha_{j} \in[0,1]$, such that

$$
\left\|x_{j}-\alpha_{j} a_{j}\right\|<\varepsilon_{n+k+1}=m(n+k)^{-1} 2^{-n-k-1} .
$$

By Remark 2,

$$
m(n+k)=\operatorname{card} A_{n+k}=m(n) \operatorname{card} A_{n+k}\left(a_{i}^{n}\right)=p m(n) .
$$

Let $y=\sum_{j=1}^{p} \lambda_{j} \alpha_{j} a_{j} \in A_{n+k}\left(a_{i}^{n}\right)^{+}$. Then from (12) and (13) we get

$$
\begin{aligned}
\|x-y\| & =\left\|\sum_{j=1}^{p} \lambda_{j}\left(x_{j}-\alpha_{j} a_{j}\right)\right\|<p \varepsilon_{n+k+1} \\
& =p m(n+k)^{-1} 2^{-n-k-1} \\
& =p(m(n) p)^{-1} 2^{-n-k-1}=m(n)^{-1} 2^{-n-k-1} .
\end{aligned}
$$

Since $y \in A_{n+k}\left(a_{i}^{n}\right)^{+}$, by (11) there is a $z \in\left[\theta, a_{i}^{n}\right]$ such that

$$
\|y-z\|<m(n)^{-1}\left(2^{-n-1}+2^{-n-2}+\cdots+2^{-n-k}\right) .
$$

Hence from (11) and (14) we get, for every $x \in A_{n+k+1}\left(a_{i}^{n}\right)^{+}$,

$$
\begin{aligned}
\left\|x-\left[\theta, a_{i}^{n}\right]\right\| & \leq\|x-z\| \leq\|x-y\|+\|y-z\| \\
& <m(n)^{-1} 2^{-n-k-1}+m(n)^{-1}\left(2^{-n-1}+\cdots+2^{-n-k}\right) \\
& =m(n)^{-1}\left(2^{-n-1}+\cdots+2^{-n-k-1}\right) .
\end{aligned}
$$

Consequently, (11) has been proved by induction. Therefore

$$
\left\|x-\left[\theta, a_{i}^{n}\right]\right\|<m(n)^{-1} 2^{-n} \text { for every } x \in \cup_{k=1}^{\infty} A_{n+k}\left(a_{i}^{n}\right)^{+} .
$$

Now, for every $x \in \hat{A}_{n+k}\left(a_{i}^{n}\right)$ and $k \in \mathbb{N}$, we have $x=\lambda_{1} x_{1}-\lambda_{2} x_{2}$, where $x_{j} \in A_{n+k}\left(a_{i}^{n}\right)^{+}$and $\lambda_{j} \in[0,1], j=1,2$, with $\lambda_{1}+\lambda_{2}=1$; see (3). Take $a_{j} \in\left[\theta, a_{i}^{n}\right]$ such that $\left\|x_{j}-a_{j}\right\|<m(n)^{-1} 2^{-n}, j=1,2$. Then we have

$$
a=\lambda_{1} a_{1}-\lambda_{2} a_{2} \in \lambda_{1}\left[\theta, a_{i}^{n}\right]-\lambda_{2}\left[\theta, a_{i}^{n}\right] \subset\left[-a_{i}^{n}, a_{i}^{n}\right],
$$


and

$$
\begin{aligned}
\|x-a\| & =\left\|\lambda_{1} x_{1}-\lambda_{2} x_{2}-\lambda_{1} a_{1}+\lambda_{2} a_{2}\right\| \\
& \leq\left\|x_{1}-a_{1}\right\|+\left\|x_{2}-a_{2}\right\|<2 m(n)^{-1} 2^{-n}=m(n)^{-1} 2^{-n+1} .
\end{aligned}
$$

Therefore

$$
\left\|x-\left[-a_{i}^{n}, a_{i}^{n}\right]\right\|<m(n)^{-1} 2^{n+1} \text { for } i=1, \ldots, m(n) .
$$

Consequently, we get the assertion by (8). The lemma is proved.

Claim 1. Using notation (8) and (10), for every $n \in \mathbb{N}$ and for every $x \in C$, there exist $x_{i} \in C_{n}\left(a_{i}^{n}\right)$, see $(8), i=1, \ldots, m(n)$, such that $x=\sum_{i=1}^{m(n)} x_{i}$.

Proof. Let $x \in C$. Take a sequence $\left\{x_{k}\right\} \subset \cup_{i=0}^{\infty} \hat{A}_{i}$ such that $x_{k} \rightarrow x$; see (6). By Remark 1, $\left\{\hat{A}_{i}\right\}$ is an increasing sequence. Therefore $\left\{x_{k}\right\} \subset \cup_{i=n+1}^{\infty} \hat{A}_{i}$ for some $n \in \mathbb{N}$. We may assume that $x_{k} \in \hat{A}_{n+n(k)}$ for every $k \in \mathbb{N}$. From (9) we get $A_{n+n(k)}=\cup_{i=1}^{m(n)} A_{n+n(k)}\left(a_{i}^{n}\right)$. Therefore, there exist $x_{i}^{k} \in \hat{A}_{n+n(k)}\left(a_{i}^{n}\right)$ and $\lambda_{i}^{k} \in[0,1], i=1, \ldots, m(n)$, such that

$$
\sum_{i=1}^{m(n)} \lambda_{i}^{k} \leq 1, \text { and } x_{k}=\sum_{i=1}^{m(n)} \lambda_{i}^{k} x_{i}^{k}
$$

From (8) we get $x_{i}^{k} \in \hat{A}_{n+n(k)}\left(a_{i}^{n}\right) \subset C_{n}\left(a_{i}^{n}\right)$ for every $k \in \mathbb{N}$ and $i=1, \ldots, m(n)$. By the compactness of $C_{n}\left(a_{i}^{n}\right)$, by passing into subsequences if necessary, we may assume that $\lambda_{i}^{k} \rightarrow \lambda_{i}$ and $x_{i}^{k} \rightarrow y_{i} \in C_{n}\left(a_{i}^{n}\right)$ for every $i=1, \ldots, m(n)$. Therefore from (15) we get

$$
x=\sum_{i=1}^{m(n)} \lambda_{i} y_{i}, \text { where } \lambda_{i} \in[0,1], i=1, \ldots, m(n), \text { and } \sum_{i=1}^{m(n)} \lambda_{i} \leq 1 .
$$

Consequently, letting $x_{i}=\lambda_{i} y_{i}$ we get $x_{i} \in C_{n}\left(a_{i}^{n}\right)$ for every $i=1, \ldots, m(n)$. The claim is proved.

From Claim 1 and from (16) it follows that

$$
C \subset \sum\left\{C_{n}(a): a \in A_{n}\right\} \text { and } C=\operatorname{conv}\left(\cup\left\{C_{n}(a): a \in A_{n}\right\}\right) \text { for every } n \in \mathbb{N} \text {. }
$$

(However, $C \neq \sum\left\{C_{n}(a): a \in A_{n}\right\}$.)

Definition 1. Let $a \in A_{n}$. We say that a non-zero point $x \in C_{n}(a)$, see (8), is maximal, if $\lambda x \notin C_{n}(a)$ for any $|\lambda|>1$.

Definition 2. We say that the expression of $x \in C$ by (16) is standard if $y_{i}$ is maximal for every $y_{i} \in C_{n}\left(a_{i}^{n}\right)$ with $y_{i} \neq \theta$.

Remark 3. It is easy to see that every $x \in C$ has a standard expression, and under Condition (i) of Theorem 2, any standard expression is unique.

Observe that, under Condition (i) of Theorem 2, the expression of $x$ in Claim 1 is unique. Therefore, we can define $P_{i}^{n}: C \rightarrow C_{n}\left(a_{i}^{n}\right), i=1, \ldots, m(n)$, by

$$
P_{i}^{n}(x)=x_{i} \text { for every } x=\sum_{i=1}^{m(n)} x_{i} \in C .
$$


Claim 2. $P_{i}^{n}: C \rightarrow C_{n}\left(a_{i}^{n}\right)$ is continuous for every $i=1, \ldots, m(n)$.

Proof. Assume that $\left\{x_{k}\right\} \subset C$ and $x_{k} \rightarrow x$. Write

$$
x_{k}=\sum_{i=1}^{m(n)} x_{i}^{k}, x=\sum_{i=1}^{m(n)} x_{i}, \text { where } x_{i}^{k}, x_{i} \in C_{n}\left(a_{i}^{n}\right) \text { for } i=1, \ldots, m(n) .
$$

We shall show that $P_{i}^{n}\left(x_{k}\right)=x_{i}^{k} \rightarrow x_{i}=P_{i}^{n}(x)$. If it is not the case, then by the compactness of $C_{n}\left(a_{i}^{n}\right)$ there exists a sequence $\{k(\ell)\} \subset \mathbb{N}$ such that $x_{i}^{k(\ell)} \rightarrow$ $y_{i} \in C_{n}\left(a_{i}^{n}\right)$ for every $i=1, \ldots, m(n)$ and that $y_{i} \neq x_{i}$ for some $i \in\{1, \ldots, m(n)\}$. Then

$$
x_{k(\ell)}=\sum_{i=1}^{m(n)} x_{i}^{k(\ell)} \rightarrow x=\sum_{i=1}^{m(n)} y_{i} .
$$

From the uniqueness of the expression of $x$ in Claim 1, we have $x_{i}=y_{i}$ for every $i=1, \ldots, m(n)$, a contradiction. The claim is proved.

Remark 4. By Claim 2, the map

$$
P_{n}=\sum_{i=1}^{m(n)} r_{a_{i}^{n}} P_{i}^{n}: C \rightarrow D_{n}=\sum_{i=1}^{m(n)}\left[-a_{i}^{n}, a_{i}^{n}\right]
$$

is continuous for every $n \in \mathbb{N}$, where $r_{a_{i}^{n}}, i=1, \ldots, m(n)$, were defined in Lemma 1. Moreover, by Lemmas 1 and 2 we have

$$
\begin{aligned}
\left\|P_{n}(x)-x\right\| & \leq \sum_{i=1}^{m(n)}\left\|r_{a_{i}^{n}}\left(x_{i}\right)-x_{i}\right\| \leq \sum_{i=1}^{m(n)} 4\left\|x_{i}-\left[-a_{i}^{n}, a_{i}^{n}\right]\right\| \\
& <4 m(n) m(n)^{-1} 2^{-n+1}=2^{n+3} .
\end{aligned}
$$

So, if $P_{n}(C) \subset C$, then $C$ is admissible, therefore, it is an AR; see [K1], [K2]. This problem seems to be easy, however we are unable to get it done. (Observe that $D_{n}$ is not contained in $C$.)

Thus, we must take a long way to the proof of Theorem 2 which involves the following characterization of ANR-spaces, established by the first author in [N1]; see also $[\mathrm{NS}]$.

\section{A characterization of ANR-SPaces}

Let $X$ be a metric space. For an open cover $\mathcal{U}$ of $X$, let $\mathcal{N}(\mathcal{U})$ denote the nerve of $\mathcal{U}$, equipped with the Whitehead topology.

Let $\left\{\mathcal{U}_{n}\right\}$ be a sequence of open covers of $X$. We say that $\left\{\mathcal{U}_{n}\right\}$ is a zero sequence iff $\sup \left\{\operatorname{diam} U: U \in \mathcal{U}_{n}\right\} \rightarrow 0$ as $n \rightarrow \infty$.

We denote $\mathcal{U}=\cup_{n=1}^{\infty} \mathcal{U}_{n}$ and $\mathcal{K}(\mathcal{U})=\cup_{n=1}^{\infty} \mathcal{N}\left(\mathcal{U}_{n} \cup \mathcal{U}_{n+1}\right)$. For every $\sigma \in \mathcal{K}(\mathcal{U})$, we write

$$
n(\sigma)=\sup \left\{n \in \mathbb{N}: \sigma \in \mathcal{N}\left(\mathcal{U}_{n} \cup \mathcal{U}_{n+1}\right)\right\} .
$$

We say that a map $f: \mathcal{U} \rightarrow X$ is a selection if $f(U) \in U$ for every $U \in \mathcal{U}$. The proof of Theorem 2 is based on the following.

Theorem 3 [N1]. A metric space $X$ is an ANR if and only if there exists a zero sequence of open covers $\left\{\mathcal{U}_{n}\right\}$ of $X$ with the following property: For any selection $g: \mathcal{U} \rightarrow X$, there exists a map $f: \mathcal{K}(\mathcal{U}) \rightarrow X$ such that if $\left\{\sigma_{n}\right\}$ is a sequence of 
simplices of $\mathcal{K}(\mathcal{U})$ for which $n\left(\sigma_{k}\right) \rightarrow \infty$, then $\operatorname{diam}\left\{f\left(\sigma_{k}\right) \cup g\left(\sigma_{k}^{o}\right)\right\} \rightarrow 0$, where $\sigma^{o}$ denotes the set of all vertices of a simplex $\sigma \in \mathcal{K}(\mathcal{U})$.

We are going to describe a sequence $\left\{\mathcal{U}_{n}\right\}$ of open covers of $C$ satisfying the conditions of Theorem 3. Using notation (10), for every $i=1, \ldots, m(n)$, we subdivide each interval $\left[-a_{i}^{n}, a_{i}^{n}\right]$ into $2 k(n)$ subintervals by $c_{0}^{i}=-a_{i}^{n}<c_{1}^{i}<\cdots<c_{2 k(n)}^{i}=a_{i}^{n}$ such that

$$
\left\|c_{j}^{i}-c_{j+2}^{i}\right\|<m(n)^{-1} 2^{-n-1} \text { for every } j=0, \ldots, 2(k(n)-1) .
$$

By Lemma 1 for every $i=1, \ldots, m(n)$, there exists a retraction $r_{i}^{n}: C_{n}\left(a_{i}^{n}\right) \rightarrow$ $\left[-a_{i}^{n}, a_{i}^{n}\right]$ such that

$$
\left\|r_{i}^{n}(x)-x\right\| \leq 4\left\|x-\left[-a_{i}^{n}, a_{i}^{n}\right]\right\| \text { for every } x \in C_{n}\left(a_{i}^{n}\right) .
$$

For every $i=1, \ldots, m(n)$ and $j=0, \ldots, 2(k(n)-1)$ we put

$$
\begin{gathered}
S_{0}^{i}(n)=\left\{x \in\left[-a_{i}^{n}, a_{i}^{n}\right]:-a_{i}^{n} \leq x<c_{2}^{i}\right\} ; \\
S_{2(k(n)-1)}^{i}(n)=\left\{x \in\left[-a_{i}^{n}, a_{i}^{n}\right]: c_{2(k(n)-1)}^{i}<x \leq a_{i}^{n} ;\right. \\
S_{j}^{i}(n)=\left\{x \in\left[-a_{i}^{n}, a_{i}^{n}\right]: c_{j}^{i}<x<c_{j+2}^{i}\right\} \text { for } j=1, \ldots, 2 k(n)-3 . \\
U_{j}^{i}(n)=\left(r_{i}^{n}\right)^{-1}\left(S_{j}^{i}(n)\right) \subset C_{n}\left(a_{i}^{n}\right) .
\end{gathered}
$$

Denote

$$
\mathbf{J}(n)=\{0, \ldots, 2(k(n)-1)\}^{m(n)} .
$$

For $\mathbf{j}=(j(1), \ldots, j(m(n))) \in \mathbf{J}(n)$, write

$$
U_{\mathbf{j}}=U_{j(1)}^{1}(n)+\cdots+U_{j(m(n))}^{m(n)}(n) \text { and } \mathcal{U}_{n}=\left\{U=U_{\mathbf{j}} \cap C: \mathbf{j} \in \mathbf{J}(n)\right\} .
$$

Our aim is to check that the sequence $\left\{\mathcal{U}_{n}\right\}$, defined by (22), satisfies the conditions of Theorem 3. First, we shall show that $\left\{\mathcal{U}_{n}\right\}$ is a zero-sequence of open covers of $C$.

Lemma 3. $\mathcal{U}_{n}$ is an open cover of $C$ for every $n \in \mathbb{N}$.

Proof. First every $U \in \mathcal{U}_{n}$ is open in $C$. In fact, let $U=U_{\mathbf{j}} \cap C \in \mathcal{U}_{n}$, where $U_{\mathbf{j}}=$ $U_{j(1)}^{1}(n)+\cdots+U_{j(m(n))}^{m(n)}(n)$. Observe that $U_{\mathbf{j}} \cap C=\cap_{i=1}^{m(n)}\left(P_{i}^{n}\right)^{-1}\left(U_{j(i)}^{i}(n)\right)$. Since $U_{j(i)}^{i}(n)$ is open in $C_{n}\left(a_{i}^{n}\right)$, see $(20)$, and since $P_{i}^{n}$ is continuous for $i=1, \ldots, m(n)$ by Claim 2, it follows that $U=U_{\mathbf{j}} \cap C$ is open in $C$.

Now, we prove that $\mathcal{U}_{n}$ covers $C$. By Claim 1 , for every $x \in C$, there exist $x_{i} \in$ $C_{n}\left(a_{i}^{n}\right), i=1, \ldots, m(n)$, such that $x=\sum_{i=1}^{m(n)} x_{i}$. Then $r_{i}^{n}\left(x_{i}\right) \in\left[-a_{i}^{n}, a_{i}^{n}\right], i=$ $1, \ldots, m(n)$. Hence, there exists $S_{j(i)}^{i}(n)$ so that $r_{i}^{n}\left(x_{i}\right) \in S_{j(i)}^{i}(n)$, whence $x_{i} \in$ $U_{j(i)}^{i}(n)$; see (19), (20). Let $\mathbf{j}=(j(1), \ldots, j(m(n))) \in \mathbf{J}(n)$; see (21). Then $x \in$ $U_{\mathbf{j}}=U_{j(1)}^{1}(n)+\cdots+U_{j(m(n))}^{m(n)}(n) ;$ see $(22)$. Consequently, $\mathcal{U}_{n}$ covers $C$, and the lemma is proved.

Lemma 4. $\left\{\mathcal{U}_{n}\right\}$ is a zero sequence: In fact, $\operatorname{diam} U<2^{-n+5}$ for every $U \in \mathcal{U}_{n}$. 
Proof. Let

$$
U=U_{\mathbf{j}} \cap C=\left(U_{j(1)}^{1}(n)+\cdots+U_{j(m(n))}^{m(n)}(n)\right) \cap C \in \mathcal{U}_{n} ; \text { see }(22) .
$$

Let $x \in U_{j(i)}^{i}(n), i=1, \ldots, m(n)$. Then, from (18) and from Lemma 2, we get

$$
\left\|x-r_{i}^{n}(x)\right\| \leq 4\left\|x-\left[-a_{i}^{n}, a_{i}^{n}\right]\right\| \leq 4 m(n)^{-1} 2^{-n+1}=m(n)^{-1} 2^{-n+3} .
$$

From $(17)$ and $(19, \mathrm{a}-\mathrm{c})$ we obtain

$$
\operatorname{diam} S_{j(i)}^{i}(n)=\left\|c_{j(i)}^{i}-c_{j(i)+2}^{i}\right\|<m(n)^{-1} 2^{-n-1} .
$$

Observe that, if $x \in U_{j(i)}^{i}(n)$, then $r_{i}^{n}(x) \in S_{j(i)}^{i}(n)$, see (20). For every $x, y \in$ $U_{j(i)}^{i}(n)$, since $r_{i}^{n}(x), r_{i}^{n}(y) \in S_{j(i)}^{i}(n)$, see $(20)$, we have

$$
\begin{aligned}
\|x-y\| & \leq\left\|x-r_{i}^{n}(x)\right\|+\left\|r_{i}^{n}(x)-r_{i}^{n}(y)\right\|+\left\|r_{i}^{n}(y)-y\right\| \\
& \leq m(n)^{-1} 2^{-n+3}+\operatorname{diam} S_{j(i)}^{i}(n)+m(n)^{-1} 2^{-n+3} \\
& <m(n)^{-1} 2^{-n+5} .
\end{aligned}
$$

Consequently

$$
\operatorname{diam} U_{\mathbf{j}} \leq \sum_{i=1}^{m(n)} \operatorname{diam} U_{j(i)}^{i}(n) \leq m(n) m(n)^{-1} 2^{-n+5}=2^{-n+5} .
$$

The lemma is proved.

The following fact is essential in our proof of Theorem 2 .

Lemma 5. Under Condition (i) of Theorem 2, if $\mathbf{j}=(j(1), \ldots, j(m(n))), \mathbf{i}=$ $(i(1), \ldots, i(m(n))) \in \mathbf{J}(n)$, see (21), such that $U_{\mathbf{j}} \cap U_{\mathbf{i}} \neq \emptyset$, then

$$
|j(k)-i(k)| \leq 1 \text { for every } k=1, \ldots, m(n) .
$$

Proof. Assume that $x \in U_{\mathbf{j}} \cap U_{\mathbf{i}}$. Then we have

$$
x=x_{j(1)}^{1}+\cdots+x_{j(m(n))}^{m(n)}=x_{i(1)}^{1}+\cdots+x_{i(m(n))}^{m(n)},
$$

where

$x_{j(k)}^{k} \in U_{j(k)}^{k}(n) \subset C_{n}\left(a_{k}^{n}\right)$ and $x_{i(k)}^{k} \in U_{i(k)}^{k}(n) \subset C_{n}\left(a_{k}^{n}\right)$ for every $k=1, \ldots, m(n)$.

From the uniqueness of the expression of $x$ in Claim 1 , we get $x_{j(k)}^{k}=x_{i(k)}^{k}$ for every $k=1, \ldots, m(n)$. Hence, from (20) we get

$$
r_{k}^{n}\left(x_{j(k)}^{k}\right) \in S_{j(k)}^{k}(n) \cap S_{i(k)}^{k}(n) \text { for every } k=1, \ldots, m(n) .
$$

Consequently,

$$
|j(k)-i(k)| \leq 1 \text { for every } k=1, \ldots, m(n) .
$$

The lemma is proved.

Remark 5. Let $U_{\mathbf{j}}=U_{j(1)}^{1}(n)+\cdots+U_{j(m(n))}^{m(n)}(n), \mathbf{j}=(j(1), \ldots, j(m(n))) \in \mathbf{J}(n)$, see (22). Then, we say that $U_{j(i)}^{i}(n)$ is the $i$-th coordinate of $U_{\mathbf{j}}$. Lemma 5 says that if $U_{\mathbf{j}}$ and $U_{\mathbf{i}}$ intersect, then the respective coordinates intersect. 


\section{Proof of Theorem 2}

Since $C$ is contractible, it suffices to prove that $C$ is an ANR. Our aim is to show that the sequence $\left\{\mathcal{U}_{n}\right\}$, defined by $(22)$, satisfies the conditions of Theorem 3 .

Let $g: \mathcal{U} \rightarrow C$ be an arbitrary selection, where $\mathcal{U}=\cup_{n=1}^{\infty} \mathcal{U}_{n}$. Then, for every $U \in \mathcal{U}$, we have $U=U_{\mathbf{j}} \cap C=\left(U_{j(1)}^{1}(n)+\cdots+U_{j(m(n))}^{m(n)}(n)\right) \cap C$, where $\mathbf{j}=$ $(j(1), \ldots, j(m(n))) \in \mathbf{J}(n)$, see $(21)$ and $(22)$. Let $g(U)=\sum_{i=1}^{m(n)} \lambda_{j(i)} x_{j(i)}$ denote the standard expression of $g(U)$, see Definition 2. Then $\lambda_{j(i)} x_{j(i)} \in U_{j(i)}^{i}(n)$ for each $i=1, \ldots, m(n)$. We define $f_{o}(U)$ by the formula

$$
f_{o}(U)=\sum_{i=1}^{m(n)} \lambda_{j(i)} r_{i}\left(x_{j(i)}\right) \in C,
$$

where $r_{i}=r_{a_{i}^{n}}, i=1, \ldots, m(n)$, see (10), were defined by Lemma 1 . By Remark 3 the standard expression is unique, hence $f_{o}$ is well-defined. Thus, we get a map $f_{o}: \mathcal{U} \rightarrow C$. (Observe that Theorem 3 does not require $f_{o}$ to be a selection.) From Lemmas 1 and 2 we get, for $U \in \mathcal{U}_{n}$,

$$
\begin{aligned}
\left\|g(U)-f_{o}(U)\right\| & =\left\|\sum_{i=1}^{m(n)} \lambda_{j(i)} x_{j(i)}-\sum_{i=1}^{m(n)} \lambda_{j(i)} r_{i}\left(x_{j(i)}\right)\right\| \\
& \leq \sum_{i=1}^{m(n)}\left\|\lambda_{j(i)} x_{j(i)}-\lambda_{j(i)} r_{i}\left(x_{j(i)}\right)\right\| \\
& \leq \sum_{i=1}^{m(n)}\left\|x_{j(i)}-r_{i}\left(x_{j(i)}\right)\right\| \leq \sum_{i=1}^{m(n)} 4\left\|x_{j(i)}-\left[-a_{i}^{n}, a_{i}^{n}\right]\right\| \\
& \leq 4 m(n) m(n)^{-1} 2^{-n+1}=2^{-n+3} .
\end{aligned}
$$

Now, using the convexity of $C$ we extend $f_{o}$ to the map $f: \mathcal{K}(\mathcal{U}) \rightarrow C$ which is linear on each simplex of $\mathcal{K}(\mathcal{U})$. Let us check that $f$ satisfies the required conditions. Let $\sigma=\left\langle U_{1}, \ldots, U_{k}\right\rangle \in \mathcal{K}(\mathcal{U})$, where $U_{1}, \ldots, U_{p} \in \mathcal{U}_{n(\sigma)}$ and $U_{p+1}, \ldots, U_{k} \in$ $\mathcal{U}_{n(\sigma)+1}$, that is,

$$
\begin{aligned}
& \sigma=\left\langle\sigma_{1}, \sigma_{2}\right\rangle \\
& \sigma_{1}=\left\langle U_{1}, \ldots, U_{p}\right\rangle \in \mathcal{N}\left(\mathcal{U}_{n(\sigma)}\right) \text { and } \sigma_{2}=\left\langle U_{p+1}, \ldots, U_{k}\right\rangle \in \mathcal{N}\left(\mathcal{U}_{n(\sigma)+1}\right) .
\end{aligned}
$$

The most important step in our proof of Theorem 2 is to estimate the diameters of $f\left(\sigma_{i}\right), i=1,2$. For the reader's convenience, we first outline the rough idea of our proof: By Remark 5, the respective coordinates of $U_{t}$ intersect. Therefore, instead of working on $U_{t}$ we can work on each coordinate of $U_{t}$. Then, using the retraction $r_{a}$ of Lemma 1, we push every coordinate into a straight line in the space. Now, observe that, because the $F$-norm $\|$.$\| of the space is monotone, the diameter of a$ finite set in a straight line in the space $X$ does not increase when taking its convex hull. So, if $\operatorname{diam} f_{o}\left(\sigma_{i}\right), i=1,2$, are really small, then $\operatorname{diam} f\left(\sigma_{i}\right), i=1,2$, are small too.

Now, we will present our arguments in details. Let $U_{t}=U_{\mathbf{j}_{t}} \cap C, t=1, \ldots, p$, with $\mathbf{j}_{t}=\left(j_{t}(1), \ldots, j_{t}(m(n(\sigma)))\right) \in \mathbf{J}(n(\sigma))$, see $(21)(22)$. Since $\cap_{t=1}^{p} U_{t} \neq \emptyset$, from Lemma 5 we get

$$
\left|j_{t}(i)-j_{t^{\prime}}(i)\right| \leq 1 \text { for every } t, t^{\prime} \in\{1, \ldots, p\} \text { and } i=1, \ldots, m(n(\sigma)) .
$$


We may assume that $j_{t}(i) \leq j_{t^{\prime}}(i)$. Then we get

$$
\left\|c_{j_{t}(i)}^{i}-c_{j_{t^{\prime}}(i)}^{i}\right\| \leq\left\|c_{j_{t}(i)}^{i}-c_{j_{t}(i)+2}^{i}\right\|<m(n(\sigma))^{-1} 2^{-n(\sigma)-1}, \text { see }(17),
$$

for every $t, t^{\prime} \in\{1, \ldots, p\}$ and $i=1, \ldots, m(n(\sigma))$. For every $i=1, \ldots, m(n(\sigma))$, we denote

$$
B_{i}=\left\{\lambda_{j_{t}(i)} r_{i}\left(x_{j_{t}(i)}\right): t=1, \ldots, p\right\} \subset\left[-a_{i}^{n(\sigma)}, a_{i}^{n(\sigma)}\right]
$$

Then we have

Claim 3. diam conv $B_{i} \leq m(n(\sigma))^{-1} 2^{-n(\sigma)+7}$ for every $i=1, \ldots, m(n(\sigma))$.

Proof. From Lemmas 1 and 2 we get, for $t=1, \ldots, p, i=1, \ldots, m(n(\sigma))$,

$$
\begin{aligned}
& \left\|\lambda_{j_{t}(i)} r_{i}\left(x_{j_{t}(i)}\right)-r_{i}\left(\lambda_{j_{t}(i)} x_{j_{t}(i)}\right)\right\| \\
& \quad \leq\left\|\lambda_{j_{t}(i)} r_{i}\left(x_{j_{t}(i)}\right)-\lambda_{j_{t}(i)} x_{j_{t}(i)}\right\|+\left\|\lambda_{j_{t}(i)} x_{j_{t}(i)}-r_{i}\left(\lambda_{j_{t}(i)} x_{j_{t}(i)}\right)\right\| \\
& \quad \leq 4\left\|x_{j_{t}(i)}-\left[-a_{i}^{n(\sigma)}, a_{i}^{n(\sigma)}\right]\right\|+4\left\|\lambda_{j_{t}(i)} x_{j_{t}(i)}-\left[-a_{i}^{n(\sigma)}, a_{i}^{n(\sigma)}\right]\right\| \\
& \quad \leq 4 m(n(\sigma))^{-1} 2^{-n(\sigma)+1}+4 m(n(\sigma))^{-1} 2^{-n(\sigma)+1} \\
& \quad=m(n(\sigma))^{-1} 2^{n(\sigma)+4} .
\end{aligned}
$$

Since $\lambda_{j_{t}(i)} x_{j_{t}(i)} \in U_{j_{t}(i)}^{i}(n(\sigma))$, we have $r_{i}\left(\lambda_{j_{t}(i)} x_{j_{t}(i)}\right) \in S_{j_{t}(i)}^{i}(n(\sigma))$, see $(20)$. Therefore, for every $t=1, \ldots, p, i=1, \ldots, m(n(\sigma))$,

$$
\left\|c_{j_{t}(i)}^{i}-r_{i}\left(\lambda_{j_{t}(i)} x_{j_{t}(i)}\right)\right\| \leq \operatorname{diam} S_{j_{t}(i)}^{i}(n(\sigma)) \leq m(n(\sigma))^{-1} 2^{-n(\sigma)-1} .
$$

It follows that

$$
\begin{aligned}
& \left\|\lambda_{j_{t}(i)} r_{i}\left(x_{j_{t}(i)}\right)-c_{j_{t}(i)}^{i}\right\| \\
& \quad \leq\left\|\lambda_{j_{t}(i)} r_{i}\left(x_{j_{t}(i)}\right)-r_{i}\left(\lambda_{j_{t}(i)} x_{j_{t}(i)}\right)\right\|+\left\|r_{i}\left(\lambda_{j_{t}(i)} x_{j_{t}(i)}\right)-c_{j_{t}(i)}^{i}\right\| \\
& \quad \leq m(n(\sigma))^{-1} 2^{-n(\sigma)+4}+m(n(\sigma))^{-1} 2^{-n(\sigma)-1} \\
& \quad<m(n(\sigma))^{-1} 2^{-n(\sigma)+5} .
\end{aligned}
$$

Consequently, from (26) we get, for $t, t^{\prime} \in\{1, \ldots, p\}$,

$$
\begin{aligned}
& \left\|\lambda_{j_{t}(i)} r_{i}\left(x_{j_{t}(i)}\right)-\lambda_{j_{t^{\prime}}(i)} r_{i}\left(x_{j_{t^{\prime}}(i)}\right)\right\| \\
& \quad \leq\left\|\lambda_{j_{t}(i)} r_{i}\left(x_{j_{t}(i)}\right)-c_{j_{t}(i)}^{i}\right\|+\left\|c_{j_{t}(i)}^{i}-c_{j_{t^{\prime}}(i)}^{i}\right\|+\left\|c_{j_{t^{\prime}}(i)}^{i}-\lambda_{j_{t^{\prime}}(i)} r_{i}\left(x_{j_{t^{\prime}}(i)}\right)\right\| \\
& \quad \leq m(n(\sigma))^{-1} 2^{-n(\sigma)+5}+m(n(\sigma))^{-1} 2^{-n(\sigma)-1}+m(n(\sigma))^{-1} 2^{-n(\sigma)+5} \\
& \quad<m(n(\sigma))^{-1} 2^{-n(\sigma)+7 .}
\end{aligned}
$$

Therefore, diam $B_{i} \leq m(n(\sigma))^{-1} 2^{-n(\sigma)+7}$. Since $B_{i}$ is contained in a straight line in $X$, see (27), from (2) we get diam conv $B_{i}=\operatorname{diam} B_{i}$. The claim is proved.

By (25) for every $x \in \sigma$ there exist $x_{i} \in \sigma_{i}$ and $\lambda_{i} \in[0,1], i=1,2$ with $\lambda_{1}+\lambda_{2}=1$, such that $x=\lambda_{1} x_{1}+\lambda_{2} x_{2}$. Then we have

$$
f(x)=\lambda_{1} f\left(x_{1}\right)+\lambda_{2} f\left(x_{2}\right) .
$$

Using Claim 3 above we obtain the following fact which provides an estimation of $\operatorname{diam} f\left(\sigma_{i}\right), i=1,2$.

Claim 4. (i) $\left\|f\left(x_{1}\right)-f_{o}\left(U_{1}\right)\right\| \leq 2^{-n(\sigma)+7}$;

(ii) $\left\|f\left(x_{2}\right)-f_{o}\left(U_{p+1}\right)\right\| \leq 2^{-n(\sigma)+6}$.

(Observe that (ii) differs from (i) because $U_{1} \in \mathcal{U}_{n(\sigma)}$; meanwhile $U_{p+1} \in \mathcal{U}_{n(\sigma)+1}$, see (25).) 
Proof of (i). Since $x_{1} \in \sigma_{1}$, we have $x_{1}=\sum_{t=1}^{p} \alpha_{t} U_{t}$, where $\alpha_{t} \in[0,1]$ and $\sum_{t=1}^{p} \alpha_{t}=1$, see (25). By the definition of $f$ and (23) we get

$$
\begin{aligned}
f\left(x_{1}\right) & =\sum_{t=1}^{p} \alpha_{t} f_{0}\left(U_{t}\right)=\sum_{t=1}^{p} \alpha_{t} \sum_{i=1}^{m(n(\sigma))} \lambda_{j_{t}(i)} r_{i}\left(x_{j_{t}(i)}\right) \\
& =\sum_{i=1}^{m(n(\sigma))} \sum_{t=1}^{p} \alpha_{t} \lambda_{j_{t}(i)} r_{i}\left(x_{j_{t}(i)}\right)
\end{aligned}
$$

Since $\sum_{t=1}^{p} \alpha_{t} \lambda_{j_{t}(i)} r_{i}\left(x_{j_{t}}(i)\right) \in \operatorname{conv} B_{i}$, see (27), it follows from Claim 3 that

$$
\begin{aligned}
\left\|f\left(x_{1}\right)-f_{o}\left(U_{1}\right)\right\| & \leq \sum_{i=1}^{m(n(\sigma))}\left\|\sum_{t=1}^{p} \alpha_{t} \lambda_{j_{t}(i)} r_{i}\left(x_{j_{t}(i)}\right)-\lambda_{j_{1}(i)} r_{i}\left(x_{j_{1}(i)}\right)\right\| \\
& \leq \sum_{i=1}^{m(n(\sigma))} \operatorname{diam} \operatorname{conv} B_{i} \\
& \leq m(n(\sigma)) m(n(\sigma))^{-1} 2^{-n(\sigma)+7}=2^{-n(\sigma)+7} .
\end{aligned}
$$

Therefore (i) holds. Similarly, we get the proof of (ii).

Using Claim 4 we can compute $\operatorname{diam} f(\sigma)$

Claim 5. $\operatorname{diam} f(\sigma) \leq 2^{-n(\sigma)+10}$.

Proof. From Lemma 4 we get

$$
\operatorname{diam} U_{1}<2^{-n(\sigma)+5} \text { and } \operatorname{diam} U_{p+1}<2^{-n(\sigma)+4} \text {, see }(25) .
$$

Since $g$ is a selection and since $U_{1} \cap U_{p+1} \neq \emptyset$ we have

$$
\begin{aligned}
\left\|g\left(U_{1}\right)-g\left(U_{p+1}\right)\right\| & \leq \operatorname{diam} U_{1}+\operatorname{diam} U_{p+1} \\
& \leq 2^{-n(\sigma)+5}+2^{-n(\sigma)+4}<2^{-n(\sigma)+6} .
\end{aligned}
$$

For each $x \in \sigma$, we write $x=\lambda_{1} x_{1}+\lambda_{2} x_{2}$, where $x_{i} \in \sigma_{i}$ and $\lambda_{i} \in[0,1]$ with $\lambda_{1}+\lambda_{2}=1$. Let $y=\lambda_{1} U_{1}+\lambda_{2} U_{p+1} \in \sigma$. Then by Claim 4 we have

$$
\begin{aligned}
\|f(x)-f(y)\| & =\left\|\lambda_{1} f\left(x_{1}\right)+\lambda_{2} f\left(x_{2}\right)-\lambda_{1} f_{o}\left(U_{1}\right)-\lambda_{2} f_{o}\left(U_{p+1}\right)\right\| \\
& \leq\left\|f\left(x_{1}\right)-f_{o}\left(U_{1}\right)\right\|+\left\|f\left(x_{2}\right)-f_{o}\left(U_{p+1}\right)\right\| \\
& \leq 2^{-n(\sigma)+7}+2^{-n(\sigma)+6}<2^{-n(\sigma)+8} .
\end{aligned}
$$

Therefore, since $\lambda_{1}+\lambda_{2}=1$, from (24) we get

$$
\begin{aligned}
\left\|f(y)-f_{o}\left(U_{1}\right)\right\| & =\left\|\lambda_{1} f_{o}\left(U_{1}\right)+\lambda_{2} f_{o}\left(U_{p+1}\right)-f_{o}\left(U_{1}\right)\right\| \\
& =\left\|\lambda_{2} f_{o}\left(U_{1}\right)-\lambda_{2} f_{o}\left(U_{p+1}\right)\right\| \leq\left\|f_{o}\left(U_{1}\right)-f_{o}\left(U_{p+1}\right)\right\| \\
& \leq\left\|f_{o}\left(U_{1}\right)-g\left(U_{1}\right)\right\|+\left\|g\left(U_{1}\right)-g\left(U_{p+1}\right)\right\|+\left\|g\left(U_{p+1}\right)-f_{o}\left(U_{p+1}\right)\right\| \\
& \leq 2^{-n(\sigma)+3}+2^{-n(\sigma)+6}+2^{-n(\sigma)+3}<2^{-n(\sigma)+7} .
\end{aligned}
$$

Consequently, from (29) we obtain

$$
\begin{aligned}
\left\|f(x)-f\left(U_{1}\right)\right\| & \leq\|f(x)-f(y)\|+\left\|f(y)-f_{o}\left(U_{1}\right)\right\| \\
& \leq 2^{-n(\sigma)+8}+2^{-n(\sigma)+7}<2^{-n(\sigma)+9} .
\end{aligned}
$$

Therefore, $\operatorname{diam} f(\sigma) \leq 2^{-n(\sigma)+10}$. The claim is proved. 
Now, we are able to complete our proof. Let $\sigma=\left\langle U_{1}, \ldots, U_{k}\right\rangle \in \mathcal{K}(\mathcal{U})$. Since $g$ is a selection and since $\cap_{i=1}^{k} U_{i} \neq \emptyset$, from Lemma 4 we get

$$
\begin{aligned}
\operatorname{diam} g\left(\sigma^{o}\right) & \leq 2 \max \left\{\operatorname{diam} U_{i}: i=1, \ldots, k\right\} \\
& =2 \max \left\{2^{-n(\sigma)+5}, 2^{-n(\sigma)+4}\right\}=2^{-n(\sigma)+6} .
\end{aligned}
$$

From (24) we obtain

$$
\operatorname{dis}\left(f(\sigma), g\left(\sigma^{o}\right)\right) \leq\left\|f_{o}\left(U_{1}\right)-g\left(U_{1}\right)\right\| \leq 2^{-n(\sigma)+3} .
$$

Therefore

$$
\begin{aligned}
\operatorname{diam}\left\{f(\sigma) \cup g\left(\sigma^{o}\right)\right\} & \leq \operatorname{diam} f(\sigma)+\operatorname{diam} g\left(\sigma^{o}\right)+\operatorname{dis}\left(f(\sigma), g\left(\sigma^{o}\right)\right) \\
& \leq 2^{-n(\sigma)+10}+2^{-n(\sigma)+6}+2^{-n(\sigma)+3}<2^{-n(\sigma)+11} .
\end{aligned}
$$

Consequently, $\operatorname{diam}\left\{f\left(\sigma_{k}\right) \cup g\left(\sigma_{k}^{o}\right)\right\} \rightarrow 0$ as $n\left(\sigma_{k}\right) \rightarrow \infty$. Hence, $C$ is an ANR by Theorem 3. Theorem 2 is proved.

Remark 6. Let us observe that Condition (ii) is not essential in the proof of Theorem 2. However, we prove Theorem 2 under this condition because it simplifies the proof and also because this condition is satisfied naturally in our application to the example of Roberts [R1].

\section{REFERENCES}

[BD] C. Bessaga and T. Dobrowolski, Some open problems on the border of infinite dimensional topology and functional analysis, Proceedings of the international conference on geometric topology, PWN, Warszawa 1980.

$[\mathrm{CDM}]$ D. Curtis, T. Dobrowolski and J. Mogilski, Some applications of the topological characterizations of the sigma-compact spaces $\ell_{f}^{2}$ nad $\Sigma$, Trans. Amer. Math. Soc. 284(1984), 837-847. MR 86i:54035

[DM] T. Dobrowolski and J. Mogilski, Problems on topological classification of incomplete metric spaces, Open problems in topology, J. van Mill and G. M. Reed (Editors) Elsevier Science Publishers B. V. North-Holland 1990. MR 92c:54001

[G] R. Geoghegan, Open problems in infinite dimensional topology, Topology Proceedings, 4(1979), 287-330. MR 82a:57015

[K1] V. Klee, Shrinkable neighbourhoods in Hausdorff linear spaces, Math. Ann. 141(1960), 281-285. MR 24:A1003

[K2] V. Klee, Leray-Schauder theory without local convexity, Math. Ann. 141(1960), 286-296. MR 24:A1004

[KM] M. G. Krein and D. P. Milman, On extreme points of regular convex sets, Studia Math. 9(1940), 133-138. MR 3:90a

[KP] N. J. Kalton and N. T. Peck, A re-examination of Roberts' example of a compact convex set with no extreme points, Math. Ann. 253(1980), 89-101. MR 82h:46055

[KPR] N. J. Kalton, N. T. Peck and J. W. Roberts, An F-space sampler, London Math. Soc. Lecture Note Series, vol. 89 Cambridge Univ. Press, 1984. MR 87c:46002

[N1] Nguyen To Nhu, Investigating the ANR-property of metric spaces, Fund. Math. 124(1984), 243-254; Correction, Fund. Math. 141(1992), 297. MR 86d:54018; MR 93k:54042

[N2] Nguyen To Nhu, The finite dimensional approximation property and the AR-property in needle point spaces, J. London Math. Soc. (to appear).

[NS] Nguyen To Nhu and Katsuro Sakai, The compact neighborhood extension property and the local equi-connectedness, Proc. Amer. Math. Soc. 121(1994), 259-265. MR 94g:54009

[NT1] Nguyen To Nhu and Le Hoang Tri, Every needle point space contains a compact convex AR-set with no extreme points, Proc. Amer. Math. Soc. 120(1994), 1261-1265. MR 94f: 54038

[NT2] Nguyen To Nhu and Le Hoang Tri, No Roberts space is a counter-example to Schauder's conjecture, Topology, 33(1994), 371-378. MR 95h:46014

[R1] J. W. Roberts, A compact convex set with no extreme points, Studia Math. 60(1977), 255-266. MR 57:10595 
[R2] J. W. Roberts, Pathological compact convex sets in the spaces $L_{p}, 0 \leq p<1$, The Altgeld Book, University of Illinois, 1976.

[Re] S. Rolewicz, Metric linear spaces, PWN, Warszawa 1972; Second publication, PWN, Warszawa 1982. MR 55:10993; MR 88i:46004a; MR 88i:46004b

Institute of Mathematics, P.O. Box 631, Bo Ho, Hanoi, Vietnam

Current address: Department of Mathematical Sciences, New Mexico State University, Las Cruces, New Mexico 88003-8001

E-mail address: nnguyen@nmsu.edu

Departamento de Geometria y Topologia, Facultad de Matematicas, Universidad Complutense de Madrid, 28040 Madrid, Spain

E-mail address: sanjurjo@sungt1.mat.ucm.es

Department of Mathematics, University of Vinh, Nghe An, Vietnam 